\title{
CHECKLIST OF THE FAMILY TENEBRIONIDAE (Coleoptera) IN Egypt
}

\author{
ELSHEWY, D. A.; M. M. SALEM and NEVEEN E. ELMETWALLY
}

Insect classification and survey Dept., Plant Protection Res. Inst., ARC, Dokki, Giza

(Manuscript received 30 November 2015)

\begin{abstract}
A $\mathrm{n}$ updated checklistof the Egyptian species of the family Tenebrionidae (Coleoptera) is presented. The listincludes about 397 species belonging to 5 subfamilies in 33 tribesbased on the records in literatures and material kept in the main reference insect collections of Egypt. Synonyms of some species and the recent taxonomic position are included. The taxa (subfamilies, tribes, genera and species) are arranged in alphabetical order.

Key words: Coleoptera ,Tenebrionidae, Egypt .
\end{abstract}

\section{INTRODUCTION}

Family Tenebrionidae (Darkling beetles) is one of the largest families of order Coleoptera, icluding about20.000known species in nearly 1700 genera of worldwide distribution (Booth et al., 1990). Ten subfamiliesare accepted now for this family (Loble et al., 2008).The majority are entirely terrestrial and very common in desert and semi- desert areas and are found in rotten wood, under logs and stones, in termite and ant nests, on plant debris, in dry dung of animals and in houses and granaries. Many are generalist feeders on detritus whether of animal or plant material and few are predators. Few species are known to be agricultural or stored products pests. The scientific names and taxonomic position of many species was a subject of taxonomic change in the last decades as a result of more critical studies such as Bouchard et al (2005, 2007, 2009 and 2011). Lawrence and Newton (2005) who provided taxonomic data on the family group names of coleoptera including Tenebrionidae with studies on biology, phylogeny and classification of some families, subfamilies with selected genera of Coleoptera.

Shalaby (1958) in the alphabetical list of the Egyptian insect in the collection of the Ministry of Agriculture, mentioned 230 tenebrionid species. Later on, El-Moursy (1996) mentioned 307 tenebrionid species of 90 genera, based on material kept in various insect collections in Egypt, although, Alfieri (1976) presented405 species within 95 genera under 25 tribes of 25 subfamilies as representing the tenebrionid fauna of Egypt. 
On the other hand, El-Akkad (2004 and 2005; a\&b); El-Metwally (2002\&2008) and ElShewy (2006 \& 2012) provided taxonomic studies, including descriptions, keys, synonyms, distribution and taxonomic notes on some Tenebrionidae of Egypt.

The present work provides a complete checklist of the currently known species of in Egypt and their taxonomic position.

\section{MATERIALS AND METHODS}

The present study is based on all records of the species of the family Tenebrionidae from Egypt, whether in literature or in the main reference insect collections in Egypt, These collections are: Collection of Ministry of Agriculture, Plant Protection Research Institute, Collection of Alfieri, Al-Azhar University, Faculty of Agriculture, Collection of Egyptian Entomological Society, Collection of Faculty of Science, Cairo University and Collection of Faculty of Science, Ain Shams University. Recent taxonomic position, senior synonyms are provided following Loble et al (2008). All taxa (subfamilies, tribes, genera and species) are alphabetically arranged.

\section{RESULTS AND DISCUSSION}

The data obtained show that there were 397 Tenebrionid species belonging to 5 subfamilies in 33 tribes with synonyms of some species and the recent taxonomic position are included as following;

\section{Alleculinae}

Alleculini

Mycetocharina megalops Fairmaire, 1894

(= Mycetocharina megalops longipennis Pic, 1923)

Mycetocharina seminigra Pic, 1925

\section{Cteniopodini}

Cteniopus gibbosus Baudi, 1877

Cteniopus priesneri priesneri Wittmer, 1936

Cteniopus priesneri luteiceps Pic, 1939

Heliotaurus angusticollis Mulsant, 1856

Heliotaurus chalybaeus Kirsch, 1869

Heliotaurus goedeli Reitter, 1890*

Heliotaurus parvicollisReitter, 1872

Omophlus gracilipes Kirsch, 1869

Omophlus lucidus Kirsch, 1869

Omophlus scutellaris Mulsant, 1856

Omophlus tenellus Kirsch, 1869 


\section{Diaperinae}

\section{Crypticini}

Cechenosternum rufulum Motschulsky, 1873

Cechenosternum rufulum nigrosuturaleKoch

Crypticussubpunctatus Walker, 1871*

Pseudoseriscius griseovestis (Fairmaire, 1879)

Pseudoseriscius maculosus murinus (Allard, 1882)

(= Crypticus maculosus murinus Allard, 1882)

(=Crypticus maculosus var mus Reitter, 1896)

\section{Diaperini}

Alphitophagus bifasciatus Say, 1824

Gnathocerus cornutus Fabricius, 1798

Platydemacaesifrons Marseul, 1876*

\section{Myrmechixenini}

Myrmechixenuspicinus Aubé,1850*

\section{Phaleriini}

Phaleriaacuminata acuminata Küster, 1852*

Phaleriaacuminata syriaca Reitter, 1916

Phaleriabimaculata Linnaeus, 1767

Phaleriabimaculata adriatica Rey, 1891

Phaleria prolixa prolixa Fairmaire, 1869

(=Phaleriaprolixa aegyptiacaSeidlitz, 1894)

Phtora aegyptaca (Reitter, 1899)

(=Cataphronetis aegyptaca Reitter, 1899)

Phtora apicilaevis (Marseul, 1876)

(=Cataphronetis apicilaevis Marseul, 1876)

Phtora subclavata (Wollaston, 1861)

(=Pseudostena subclavata Wollaston, 1861)

\section{Trachyscelini}

TrachyscelisaphodioidesLatreille, 1809

(= Trachyscelisaphodioides subopacus Pic, 1923)

Trachyscelistenuistriatus Fairmaire, 1886

\section{Lagriinae}

Belopini

Centorus aegyptiacus (Zoufal, 1893)

(=Belopus aegyptiacus Zoufal,1893)

Centorus heydeni Zoufal, 1893*

Centorus microceps (Motschulsky, 1872)

(=Belopus microceps Motschulsky, 1872) 


\section{Cossyphini}

Cossyphus moniliatus Reitter, 1917

Cossyphus moniliferus Chevrolat, 1833

\section{Pimeliinae}

\section{Adelostomini}

Adelostoma abyssinicum hirsutum Koch, 1935

Adelostoma cordatum Solier, 1837

Adelostoma grande Hagg, 1879

Adelostoma sulcatum sulcatum Duponchel, 1827

(=Adelostoma sulcatum deplanatum Haag, 1875)

(=Adelostoma sulcatum grandiformis Koch, 1935)

Machlopsiscrenatacostata Redtenbacher, 1868

Machlopsis pseudoalata Gridelli, 1930

Pogonobasis ornata Solier, 1837

\section{Adesmiini}

Adesmia antiqua antiqua Klug,1830

(=Adesmia antiqua aethiopica Allard, 1885)

Adesmia cancellata abbreviata Klug, 1830*

Adesmia cancellata cancellata Klug, 1830

Adesmia cancellata clathrata Solier, 1835*

Adesmiacarinatacarinata Solier, 1835

(=Adesmia elevata Solier, 1835)

Adesmia cothurnata Forskal, 1775

(=Adesmia bicarinata Klug, 1830)

(=Adesmia bicarinata glabrior Schatz\&Koch, 1934)

Adesmia coucyi Lucas, 1850*

Adesmia dilatata affinis Solier, 1835

(=Adesmia solieri Lucas, 1844)

Adesmia dilatata dilatata Klug, 1830

(=Adesmia dilatata drakei Crotch, 1872)

Adesmia lacunosa Klug, 1830

Adesmia longipes Fabrcius, 1775

Adesmia metallica Klug, 1830

(=Adesmia faremonti Lucas, 1844)

(=Adesmia metallica var. chakouri Reitter, 1916)

Adesmia metallica brozai Ardoin, 1978*

Adesmia metallica laevior Ardoin, 1978*

Adesmia metallica syriaca Baudi, 1874

Adesmia montana acervata Klug, 1830 
Adesmia montana minor Gridelli, 1930

Adesmia montana montana Klug, 1830

Adesmia montana parallela Miller, 1861

Adesmia monilis monilis Klug, 1830

Adesmia monilis pluriseriata Schuster, 1928

Adesmia reticulata (Klug, 1830)

Adesmia reticulatabasimargo Reitt, 1916.

Adesmia tenebrosa Solier, 1835*

\section{Akidini}

Akis trilineata Herbst, 1799

(=Akis barbara Solier, 1837)

Akiscyrenaica Schuster, 1928

(=Akis reflexa cyrenaicaSchuster, 1928)

Akis elevata elevata Solier, 1836

Akis gestroi Schuster, 1922

(=Akis reflxeagestroi Schuster, 1922)

Akis latreillei Solier, 1836

Akis reflexareflxea Fabricius, 1775

Akissculptior Koch, 1935

(=Akis spinosaLinnaeus, 1764)

(=Akiselevata var. sculptior, Koch, 1935)

Moricacostitubera (Marseul, 1883)

(=Akiscostitubera Marseul, 1883)

Moricagrossa Linnaeus, 1767

\section{Asidini}

Alphasidaauriculata Solier, 1836

(= Alphasidamystica Wilke, 1921)

\section{Bromorphini}

Boromorphus aegyptiacus Reitter, 1908

\section{Cnemeplatiini}

Cnemeplatia atropos A. Costa, 1847

Philhammus aharonii Reitter, 1910

(=Philhammus andresi Koch, 1935)

Philhammus sericans Fairmaire, 1871*

\section{Erodiini}

Amnodeis confluens Miller, 1858

(= Amnodeis arabicus Ritter,1914)

Amnodeis giganteeus Reiche \& Saulcy, 1857

Apentanodes globosus Reiche \& Saulcy, 1857 
Arthrodeiscrypticoides Fairmaire,1879

Arthrodeis rotundatus Solier, 1834

(=Arthrodeis rotundatus var. gebieni Reitter, 1914)

(=Arthrodeis cruciatus Solier, 1834)

Erodius bicostatus Solier, 1834

(= Erodiustripolitance Molder, 1943)

Erodius bilineatus Olivier, 1791

Erodius costatus Solier, 1834

Erodius dejeanii Solier, 1834

Erodius duponchelii Allard, 1865

(=Erodius dejeanii var. duponchelii Allard, 1865)

(=Erodius lineolatusReitter, 1914)

Erodius gibbus Fabricius, 1775

(=Erodius syriacus Solier, 1834)

Erodius glabratus Solier, 1834

Erodius kneuckeri Andres, 1920

Erodius kneuckeri semisculptus Koch, 1935

Erodius krugeri Schuster, 1928

Erodius nitidicollis Solier, 1834

(= Erodius pulverulentus Allard, 1873)

Erodius octocostataus Peyerimhoff, 1907

Erodius opacus Kraatz, 1865

Erodius orientalis brevicostatus Solier, 1834

(= Erodius dimidiatipennis Kraatz, 1865)

Erodius puncticollisSolier, 1834

(= Erodius contractus Kraatz, 1865)

Erodius puncticollis maillei Solier, 1834

Erodius puncticollis sinaiticus Crotch, 1872

Erodius quadrilineatus Kraatz, 1865

Erodius zophosoides Allard, 1865

\section{Pimeliini}

Pachylodera brevicornis Quedenfeldt, 1890

Pimelia aegyptiaca Andres, 1929

(= Pimelia nilotica aegyptiaca Andres, 1929)

Pimelia angulata Fabricius, 1775

Pimelia angulata antiaegypta Koch, 1937

Pimelia angulosa Olivier, 1795

Pimelia arabica Klug, 1830

Pimeliaarabicola Schatzmayer \& Koch, 1934 
(=Pimelia canescens arabicola Schatzmayer \& Koch, 1934)

Pimelia barthelemyi Solier, 1836

Pimelia böhmi Reitter, 1915

Pimelia bottae Sénac, 1887

Pimelia canescns Klug, 1830

(= Pimelia depilate Solier, 1836)

Pimelia comata Klug, 1830

(=Pimelia ornata Miller, 1861)

Pimelia confalonierii Gridelli, 1930

(=Pimelia consobrina Confalonieri Gridelli, 1930)

Pimelia senegalensis Olivier, 1795

(=Pimelia cultimargo Sénac, 1884)

Pimelia denticostata Koch, 1935

(= Pimelia grandis var. denticostata Koch, 1935)

Pimelia derasa Klug, 1830

Pimelia grandisklug, 1830

(= Pimelia sudanica Fairmair, 1882)

Pimelia granulata Solier, 1836

(=Pimelia doumeti Sénac, 1882)

(=Pimelia lucasii Reiche, 1861)

Pimelia hirtella Sénac, 1887

Pimelia latrillei Solier, 1836

(=Pimelia latrillei permixta Sénac, 1881)

Pimelia interpunctata (Klug, 1830)

(=Doderoellainterpunctata cyrenaica Suster, 1926)

(=Doderoella interpunctata interpunctata Klug, 1830)

(=Pimelia carinata Solier, 1836)

Pimelia letourneuxi Sénac, 1880

Pimelia mittrei Solier, 1836

Pimelia nilotica Sénac, 1884

(= Pimelia angulata nilotica Sénac, 1884)

Pimelia priesneri Koch, 1935

Pimelia raffrayi Sénac, 1882

Pimelia sericea Olivier, 1795

Pimelia sinaitica Schatzmayer \& Koch, 1934

(= Pimelia angulate sinatica Schatzmayer \& Koch, 1934)

Pimelia spinulosa Klug, 1830

Pimelia subquadrata Sturm, 1826

(=Pimelia irrorata Solier, 1836) 
Pimelia tenuicornis Solier, 1835

(=Pimelia granulata tenuicornis Solier, 1836)

Pimelia tenuitarsis Reitter, 1915

Pimelia theveneti Sénac, 1880

Pimelia tripolitana Sénac, 1884*

(= Pimelia tenuicornis var. tripolitana Sénac, 1884)

Pimelia urticata Klug, 1930

(= Pimelia urticata exanthematica Klug, 1830)

Prionotheca coronata Olivier, 195

Pterolasia squalid (Solier, 1836)

(= Thriptera lanata Peyerimhoff, 1907

(= Thriptera boehmi Reitter, 1908)

Storthocnemis abyssinica(Hagg, 1876)

(=Leucolaephus latifrons Fairmaire, 1882)

Storthocnemis steckeri Karsch, 1881

Trachyderma andresi Gridelli 1928

Trachyderma emondi(Solier, 1836)

(=Ocnera longicollis Desbrochers, 1881)

Trachyderma genei (Solier, 1836)

(=Ocnera habelmannKraatz, 1865)

Trachyderma hispida (Forskal, 1775)

(= Tenebrio hispida Forskal, 1775)

(=Ocnera hispida latreillei Solier, 1836)

Trachyderma leprieuri (Allard, 1886)

(=Ocnera sparsispina Boehm, 1914)

Trachydermalima(Petagna, 1819)

(=Ocnera angustata Solier, 1836)

Trachyderma major (Baudi, 1875)

(=Ocnera hispida var. major, Baudi, 1875)

Trachyderma parvicollis Baudi, 1875

Trachyderma philistina Reiche - Saulcy, 1857

Thriptera crinita klug, 1830

(=Thriptera maillei, Solier, 1836)

(=Ocnera pygmaea, Miller, 1861)

Thriptera kraatzi Hagg, 1876

(= Thriptera sororcula Reitter, 1894)

Thripteraminuta (Pic, 1897)

(=Micipsa minuta Pic, 1897)

Thriptera pilipes Kraatz, 1865 


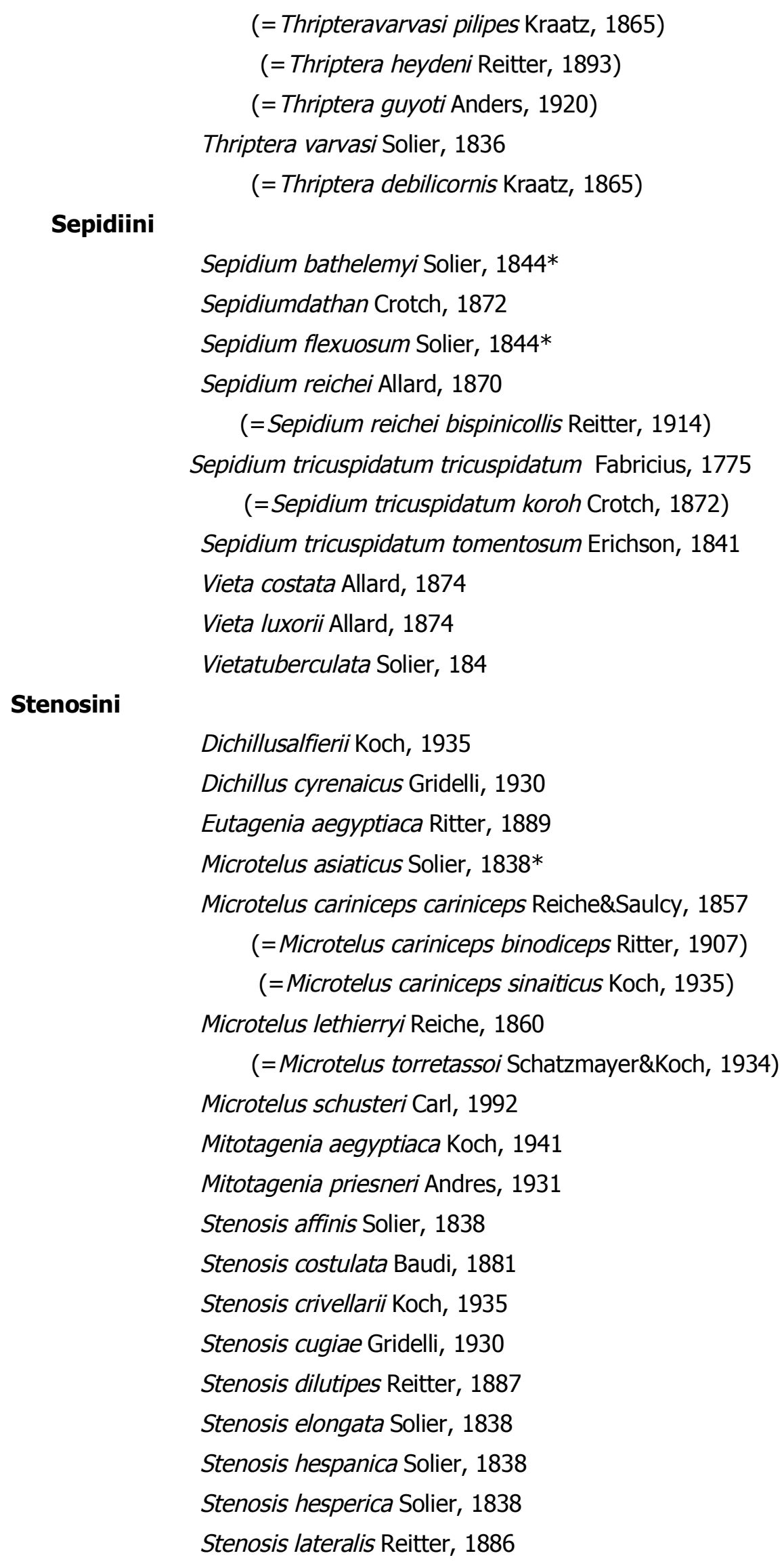

\section{Sepidiini}

Sepidium bathelemyi Solier, 1844*

Sepidiumdathan Crotch, 1872

Sepidium flexuosum Solier, 1844*

Sepidium reichei Allard, 1870

(=Sepidium reichei bispinicollis Reitter, 1914)

Sepidium tricuspidatum tricuspidatum Fabricius, 1775

(=Sepidium tricuspidatum koroh Crotch, 1872)

Sepidium tricuspidatum tomentosum Erichson, 1841

Vieta costata Allard, 1874

Vieta luxorii Allard, 1874

Vietatuberculata Solier, 184

\section{Stenosini}

Dichillusalfierii Koch, 1935

Dichillus cyrenaicus Gridelli, 1930

Eutagenia aegyptiaca Ritter, 1889

Microtelus asiaticus Solier, 1838*

Microtelus cariniceps cariniceps Reiche\&Saulcy, 1857

(=Microtelus cariniceps binodiceps Ritter, 1907)

(=Microtelus cariniceps sinaiticus Koch, 1935)

Microtelus lethierryi Reiche, 1860

(=Microtelus torretassoi Schatzmayer\&Koch, 1934)

Microtelus schusteri Carl, 1992

Mitotagenia aegyptiaca Koch, 1941

Mitotagenia priesneri Andres, 1931

Stenosis affinis Solier, 1838

Stenosis costulata Baudi, 1881

Stenosis crivellarii Koch, 1935

Stenosis cugiae Gridelli, 1930

Stenosis dilutipes Reitter, 1887

Stenosis elongata Solier, 1838

Stenosis hespanica Solier, 1838

Stenosis hesperica Solier, 1838

Stenosis lateralis Reitter, 1886 
Stenosis lateralis bogosensis Reitter, 1886*

Stenosis parvicollis Desbrochers, 1881

Stenosis pubescens Solier, 1838

Stenosis torretassoi Koch, 1935

Tagenostola seriepilosa mulleri Reitter, 1886

\section{Tentyriini}

Cyphostethe heydeni Hagg- Rutenberg, 1877

(=Cyphostethe saharensis Chobaut, 1897)

Dialognatha crenata Reiche \& Saulcy, 1857

Fourtaus brevicornis Pic, 1920

Hegeterocara arabica Reitter, 1900

Hionthis angusticollis Schuster, 1920

Hionthis tentyrioides Miller, 1861

Hyperops tagenoides aegyptiaca Kraatz, 1865

Imatismus villosus (Haag- Rutenberg, 1870)

(=Curimosphena villosusHaag - Rutenberg, 1870)

Imatismus pavesii(Gestro, 1892)

$$
\text { (=Curimosphena pavesii Gestro) }
$$

Mesostena angustata Fabricius, 1775

(= Mesostena elegans Solier, 1853)

(= Mesostena punctate Eschscholtz, 1831)

Mesostena klugii Solier, 1835

Mesostena puncticollis Solier, 1835

Mesostenopa aegyptiaca Koch, 1940

Mesostenopa cavatica Andres, 1926

(=Mesostenopa longicornis cavatica Andres, 1926)

Mesostenopa habessinica habessinica Kraatz, 1865

Mesostenopa longicornis Kraatz, 1865

Mesostenopa picea Kraatz, 1865

Mesostenopa picea agilis Gestro, 1881

Mesostenopa piceasinaitica Schatzmayer \& Koch, 1934

Mesostenopa peyerimhoffi Gridelli, 1938

(=Mesostenopa gracilis Peyerimhoff, 1907)

Micipsa douelucas, 1856

(=Micipsa grandis Kraatz, 1865)

Micipsa philistine Reiche ,Saulcy, 1857

Micipsa schaumii Kraatz, 1856

Oterophloeus alveatus peyerimhoffi Koch, 1935

Oterophloeus deflexangulus Reitter, 1900 
Oterophloeusdesioi divinus Koch, 1935

Oterophloeushaagi haagi Kraatz, 1865

Oterophloeushaagi orientalis Koch, 1935

Oxycara ardoini Kaszab, 1979*

Oxycara deronectinum Reitter, 1900

Oxycara ferreri Lillig, 2001*

Oxycara hegetericum Reiche ,Saulcy, 1857

Oxycara occidentale Lillig,2001*

Oxycara peyerimhoffi Lillig, 2001*

Oxycara productum Peyerimhoff, 1907

Oxycara pumilumReitter, 1900*

Oxycara pygmaeumReiche, Saulcy, 1857

Oxycara subcostatum Guérin, 1862

Phaeotribon pulchellus Kraatz, 1865

Prochoma audouini Solier, 1835*

Psammoica lucida Solier, 1835

Rhytinota praelonga Reiche, 1850

Scelosodiscastaneus Eschscholtz, 1831

Schweinfuthia alfierii Schatzmayer \& Koch,1934

Schweinfuthia sinaitica Andres, 1922

Tentyria duponti Solier, 1835

Tentyria glabra Fabricius, 1775

Tentyria longicollis Lucas, 1855

Tentyria mauritanica Baudi, 1875*

Tentyria mucronata Steven, 1829*

Tentyria platyceps Steven, 1829*

(= Tentyria calcarata Reitter, 1900)

Tentyria punctatastriata Solier, 1835

Tentyria sinaitica Peyerimhoff, 1907

Tentyrina duplicata duplicata Reitter, 1900.

(= Tentyrina boehmi duplicata Reitter)

Tentyrina duplicata boehmi Reitter, 1909

(= Tentyrina boehmi boehmi Reitter)

Tentyrina duplicata schusteri Schatz.\&Koch, 1934.

(= Tentyrina boehmi Schusteri Schatz.\&Koch)

Tentyrina duplicata brachythorax Gridelli, 1929

Tentyrina duplicata manzonii Gridelli, 1929

Tentyrina orbiculata orbiculata Fabricius, 1775

Tentyrina orbiculata subsulcata Reiche\& Saulcy, 1857 
Tentyrina palmeri Crotch, 1872

Thraustocolus leptoderus Kraatz, 1865

Thraustocolus montandoni Schuster, 1915

Thraustocolus priesneri Koch, 1934

Trichosphaena perraudieri Marseul, 1867

\section{Zophosini}

Zophosis abbreviata Solier, 1834

(=Zophosis semilineata Deyrolle, 1867)

Zophosis bicarinata quadricostata Solier, 1834

Zophosis complanata Solier, 1834

(=Zophosis complanata deplanata Böhm, 1909)

(=Zophosis pulverulenta Deyrolle, 1867)

Zophosis dilatata dilatata Deyrolle, 1867

Zophosis farinosaBlair, 1914

Zophosis lethierryi Deyrolle, 1867*

Zophosis marseuli Deyrolle, 1867

Zophosis minuta Fabricius, 1775

Zophosis nigroaenea Deyrolle, 1867

(=Zophosis mozabita Fairmaire, 1897)

Zophosis oxycaroides Koch, 1935

Zophosis parallela Miller, 1861

Zophosis personataErichson, 1841

Zophosis pfeifferiGredler, 1877

Zophosis pharaonisReitter, 1916

Zophosis plana plana Fabricius, 1775

(=Zophosis plana elongata Koch, 1935)

(=Zophosis approximata Deyrelle, 1867)

(=Zophosis astacura Peyerimhoff, 1931)

(=Zophosis carinata Solier, 1834)

Zophosis posticalisDeyrolle, 1867

Zophosis punctata punctata Brullé, 1832

(=Zophosis punctata algerianaSolier, 1834)

(=Zophosis punctata ovate Brullé, 1832)

Zophosis punctata pygmaeaSolier, 1834

Zophosis quadrilineata Olivier, 1791

Zophosis rotundata Ménétriés, 1849

Zophosis sulcata Deyrolle, 1867

(=Zophosis bohemanni Deyrolle, 1867)

(=Zophosis maeklini Deyrolle, 1867) 
Zophosis trilineata Olivier 1795

Zophosis viridilimbata Chobaut, 1899

(=Zophosis Cyrenaica Schuster, 1928)

\section{Tenebrioninae}

\section{Alphitobiini}

Alphitobius diaperinusPanzer, 1796

Alphitobius laevigatus Fabricius, 1781

\section{Blaptini}

Blaps andresKoch, 1935

(=Blaps bifurcata andresi Koch, 1935)

Blaps approximans Seidlit, 1893

Blaps bifurcate Solier, 1848

Blaps cognata Solier, 1848

Blaps cordicollis Solier, 1848

Blaps doderoi Shuster, 1922*

Blaps gigas Linné, 1767*

(= Blapsproduct Brullé, 1832)

Blaps juliae Allard, 1880

Blaps kollari Seidlitz, 1893

Blaps laticollis Solier, 1848

Blaps nitens Laporte, 1840*

Blaps nitens laportei Ardoin, 1973*

Blaps nitens barbara Solier, 1848*

Blaps pharao Seidlit, 1893

Blaps planaSolier, 1848

Blaps planicollis Laporte, 1840

Blaps polychresta Forskscal, 1775

(=Blaps sulcate Fabricius, 1775)

Blaps propheta Reiche, 1861*

(= Blaps algeriaca Ballion, 1888)

Blaps schweinfurthi Seidlitz, 1893

Blaps sulcata Laporte, 1840

Blaps sulcifera Seidlitz, 1893

Blaps taeniolata Ménétriés, 1832

Blaps tingitana antennalis Allard, 1880*

Blaps tingitana tingitana Allard, 1880*

Blaps tripolitanica Karsch, 1881

Blaps wiedemanni Solier, 1848 


\section{Helopini}

Helopinini

Catomus consentaneus Küster, 1851

Catomus subparallelus Koch, 1935

Catomus torretassoi Koch, 1935

Entomogonus angulicollis Mulsant \& Wachanru, 1853

Odocnemis clarus Allard , 1876*

Xanthomus cyprius Grimm , 1991*

Xanthomus pallidus (Curtis, 1830)

(=Cylindronotus pallidus Curtis, 1830)

Drosochrus costatus (Solier, 1848)

(=Helopinus costatus Solier, 1848)

Drosochrus costatus aegyptiacus (Gridelli, 1939)

(=Helopinus costatus aegyptiacus Gridelli, 1939)

Melanimini

Cheirodes asperulus (Reitter, 1884)

(=Anemia aegyptiacus Pic, 1936)

(=Anemia asperula seriesetosa Baudi, 1894)

Cheirodes brevicollis (Wollaston, 1864)

(=Anemia aphodioides Walkaston, 1864)

(=Anemia fausti Solsky, 1881)

Cheirodes pilosus (Tournier, 1868)

(=Anemia pilosus Tournier, 1868)

Cheirodes sardous (Gené, 1839)

(=Anemia sardous Gené, 1839)

Cheirodes submetallica (Raffray, 1873)

(=Anemia submetallica Raffray, 1873)

\section{Opatrini}

Ammobius rufus Lucas, 1846

Brachyesthes chrysomelina Costa, 1878

Brachyesthes gastonis Fairmaire, 1873

Brachyesthes pilosellaMarseul, 1867

Caedius cassidoides Fairmaire, 1894

Clitobius oblongiusculus oblongiusculus Fairmaire, 1875

Clitobius oblongiusculus schneideri Allard, 1882

Clitobius ovatus Erichson, 1843

Cyptus aegyptiacus(Mulsant \& Ray, 1859)

(=Caedius aegyptiacus Mulsant\& Ray, 1859)

Dilamus pictusBaudi, 1881 
Dilamus planicollis Fairmaire, 1883

Diphyrrhynchus aenescons Fairmaire,1892

Eurycaulus dubius Gridelli , 1930

Eurycaulus granulatus Reitter, 1904

Eurycaulus henoni Fairmaire, 1897

Eurycaulushirsutus Miller, 1861

Eurycaulus hirustus haliopolis Koch, 1935

Eurycaulus peyerimhoffi Reitter , 1904

Gonocephallum controversum Gridelli, 1948

Gonocephallum patruele Erichson, 1843

Gonocephalumperplexum Lucas, 1846

Gonocephalumprolixum Erichson, 1843

Gonocephallum rusticum Olivier, 1811

Gonocephallum setulosum Faldermann, 1837

Gonocephallum soricinum Reiche \& Saulcy, 1857

Gonocephalum strigosum Reiche, 1850

Mesomorphus setosus Mulsant \& Ray, 1853

Neopachypterusniloticus (Miller, 1861)

(=Pachypterusniloticus Miller, 1861)

Opatroides punctulatusBrullé, 1832

Opatropis affine Billerg, 1815

(=Opatropis hispidum Brullé, 1838

Opatrum obesum Olivier, 1811

Opatrum porcatum Fabricius, 1792

Penthicus viberti (Reitter, 1906)

(=Lobothorax viberti Reitter, 1906)

Platynosum paulinae Mulsant \& Ray, 1859

Prodilamus bohmi (Reitter, 1904)

(=Dilamus bohmi Reitter, 1904)

Prodilamus ferrantei (Reitter, 1908)

(=Dilamus ferrantei Reitter, 1908)

Proscheimus arabicus Desbrochers, 1881

Sclerum armatum (Waltl, 1835)

(=Scleron armatum Waltl, 1835)

Sclerum dubium(Gridelli, 1930)

(=Scleron dubium Gridelli, 1930)

Sclerum multistriatum (Forskal, 1775)

(=Scleron multistriatum Forskal, 1775)

Sclerum orientalis (Fabricius, 1775) 
(=Sclerom orientalis Fabricius, 1775)

Sclerum sudanicum (Koch, 1935)

(=Scleron sudanicum Koch, 1935)

Sclerum sulcatum saharense (Peyerimhoff, 1930)

(=Scleron sulcatum saharense Peyerimhoff, 1930)

Scleropatroides hirtulus (Baudi, 1876)

(=Scleropatrum hirtulus Baudi, 1876)

Palorini

Palorus depressus Fabricius , 1790

Palorus ficicolaWollaston, 1867

(= Palorus deserticola Sahlberg, 1913)

(=Palorus subfilum Fleischer, 1900)

Palorus ratzeburgii Wissmann, 1848

Palorus subdepressus Wollaston, 1864

Pedinini

Cabirutus cyrenaicus Gridelli, 1930

Cabirutus rotundicollis Miller, 1861

Cabirutus simonis Reitter, 1904

Dendarus calcaroides Peyerimhoff, 1907

Dendarus piceus Olivier, 1811

Dendarus syriacus Reichr \& Saulcy, 1857

Leichenum muelleri Gridelli, 1939

Leichenum pulchellum Lucas, 1846

Leichenum pulchellum pumilum Baudi, 1876 *

Pedinus olivieri Mulsant \& Rey, 1853

Zidalus corvinus (Mulsant \& Rey, 1853)

(=Opatrinuscorvinus Mulsant \& Rey, 1853)

Zidalus erythraeus Gridelli, 1940*

Zidalus niloticus (Mulsant \& Ray, 1853)

(=Opatrinus niloticus Mulsant \& Ray, 1853)

\section{Scaurini}

Scaurus aegyptiacus Solier, 1838

Scaurus atratus Fabricius, 1775

Scaurus bougoni Fairmaire, 1883

Scaurus carinatus Solier, 1838

Scaurus puncticollisSolier, 1838

Scaurus puncticollis macricollis Allard, 1882

Scaurus puncticollis syriacus Reitter, 1914

Scaurus sanctiamandi Solier, 1838* 
Scaurus varvasi Solier, 1838*

Tenebrionini

Tenebrio molitor Linnaeus, 1758

Tenebrio obscurus Fabricius, 1792

Tenebrio syriacus Zoufal, 1892

Triboliini

Latheticus oryzae Waterhouse, 1880

Tribolium castaneum Herbst, 1791

Tribolium confusum JacquelinDuval, 1861

Tribolium madens Charpentier, 1825*

Ulomini

Cenoscelis pulla (Erichson, 1843)

(=Uloma pulla Erichson, 1843)

(=Eutochia pullaErichson, 1843)

*Species mentioned in Loble et al. (2008) as recorded in Egypt.

\section{REFERENCES}

1. Alfieri, A. 1976. The Coleoptera of Egypt (monograph). Mem.Soc. Ent. Egypt, 5; Tenebrionidae: 166-202.

2. Booth, R.G.; M. L. Cox; and R.B. Madge. 1990.IIE Guides to insects of importance to man, 3. Coleoptera: 100 - 105.

3. Bouchard, P.; J.F. Lawrence; A.E. Davies and A.F. Newton. 2005. Synoptic classification of the world Tenebrionidae (Insecta: Coleoptera) with A review of family-Group Names. Ann. Zool. (Warszawa), 55(4): 499-530.

4. Bouchard P.; V.V. Grebennikov; A.B.T. Smith.; H. Douglas. 2009. Biodiversity of Coleoptera [pp. 265-301]. In: Foottit RG, Adler PH (Eds). Insect biodiversity: science and society. Blackwell Publishing, Oxford, $656 \mathrm{pp}$

5. Bouchard P.; I. Löbl; O. Merkl. 2007. Nomenclatural notes ontenebrionid beetles of the Palaearctic Region (Insecta: Coleoptera). Annales Zoologici (Warszawa) 57 (3): 385-394.

6. Bouchard P.; Y. Bousquet; A.E. Davies; M.A. Alonso-Zarazaga; J.F. Lawrence; C.H.C. Lyal; A.F. Newton; C.A.M. Reid; M. Schmitt; S.A. Ślipiński; A.B.T. Smith. 2011. Family-group names in Coleoptera (Insecta). ZooKeys 88: 1-972.

7. El-Akkad, M.K. 2004. A Taxonomic Review of Genus Adesmia Fischer (Pimiliinae: Tenebrionidae: Coleoptera) From Egypt. Egypt.J. Appl. Sci; 19(3): 271-297. 
8. El-Akkad, M.K. 2005a. Key to species and subspecies of Genus Zophosis L. of Egypt (Tentyriinae: Tenebrionidae: Coleoptera). Egypt.J. Appl. Sci; 20 (2): 268277.

9. El-Akkad, M.K. 2005b. Revision of Tribe Erodiini (Pimelliinae: Tenebrionidae: Coleoptera) from Egypt.Egypt.J. Appl. Sci; 20 (2): 240-267.

10. EL-Metwally, N.E. 2002. Taxonomic study of subfamily Tentyrinae (Family Tenebrionidae - Order Coleoptera) in Egypt, M.sc., Faculty of Science, Ain Shams Uni.: 265 pp.

11. EL-Metwally,N.E. 2008. Faunistic and Taxonomic studies on the subfamilies Akidinae, Pimeliinae and Blaptinae (Family: Tenebrionidae, Order: Coleoptera) in Egypt. P.hD., Faculty of Science, Ain Shams Uni.: 404 pp.

12. El-Moursy, A.A.; H.H. Fadl and M.K. El-Akkad. 1996. Biological diversity of Egypt (Col.). United Nat. Env.Prog. Nat. Nial. Unit, 4: 1-341 \& 5: 1-708.

13. El-Shewy, D.A.L. 2006. Taxonomic studies on Subfamilies Opatrinae and Pedininae (Family Tenebrionidae - Coleoptera) in Egypt, M.Sc. Thesis, Entomology Department, Faculty of Science, Al-Azhar University: Egypt; 258 pp.

14. El-Shewy, D.A.L. 2012. survey and classification of some species of familytenebrionidae (Order Coleoptera) from Egypt. P.hD. Thesis, Entomology Department, Faculty of Science, Al-Azhar University: Egypt; 501 pp.

15. Lawrence, J. F. and A. F. Newton. 2005. Families and subfamilies of Coleoptera (with selected genera, notes, references and data on family-group names) [pp. 779-1006]. In: Pakaluk J, Ślipiński SA (Eds) Biology, phylogeny and classification of Coleoptera: Papers celebrating the 80th birthday of Roy A Crowson. Vol II. Muzeum Instytut Zoologii PAN, Warszawa, x + 1092 pp. in 2 vols. [31 Mar 1995 (teste S. A. Ślipiński)]

16. Löbl I.; O. Merkl; K. Ando; P. Bouchard; M. Lillig; K. Masomuto; W. Schawaller. 2008. Catalogue of Palaearctic Coleoptera. Volume 5.Tenebrionoidea.Apollo Books, Stenstrup, 670 pp. [publ. 15 Apr 2008 (verso of title page)].

17. Shalaby, F. (1974 - 1979). survey and biological control studies on the dung beetles in Egypt. Final report. Project no. Eg-Ars-14 : 603. 


\title{
قائمة مرجعية من أسرة تينيبريونيدى ( خنافس الظلام) TENEBRIONIDAE
}

\author{
داليا عبد اللطيف الثيوى ، مجدى محمد سالم ، نيفين عصام المتولى \\ - قسم التصنيف - معهد بحوث وقاية النباتات - مركز البحوث الزراعبة - دقى - جيزة
}

يقدم هذا العمل قائمة مرجعية محدثة للأنو اع المصرية من فصيلة تنيييريونيدى (خنافس الظلام) التابعة لرتبة الحشر ات غمدية الأجنحة .

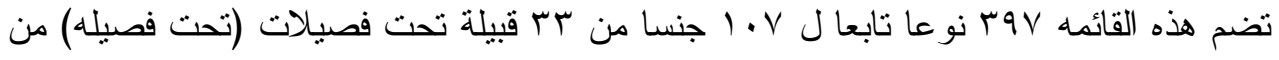
فصيلة تينيبريونيدى ، وهى تمنل الفونه الحشرية المصرية من هذه الخنافس .

تستتد هذه القائمة على التسجيلات المذكورة فى القو ائم و الكتالوجات و المر اجع التصنيفية المحلية

و العالمية وايضا تستتد على النماذج المدرجه فى المجموعات الحشرية المرجعية فى مصر .

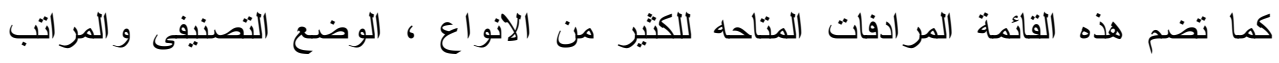
التصنيفية المذكوره وفقا لأحدث الكتالوجات و الدر اسات التصنيفيه الحديثه .

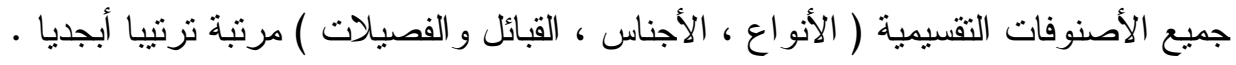

